\title{
Đánh giá những khó khăn riêng biệt của việc dạy và học các môn Toán trực tuyến trình độ đại học
}

\section{Evaluating the challenges in teaching and learning Mathematical online courses}

\author{
Phạm Thị Thu Hoa ${ }^{1 *}$, Phạm Thị Thu Hường ${ }^{1}$ \\ ${ }^{1}$ Trường Đại học An Giang, Trường Đại học quốc gia Thành phố Hồ Chí Minh, Việt Nam \\ *Tác giả liên hệ, Email: ptthoa@agu.edu.vn
}

THÔNG TIN

DOI: $10.46223 /$ HCMCOUJS. soci.vi.16.1.1425.2021

Ngày nhận: 30/01/2021

Ngày nhận lại: 19/03/2021

Duyệt đăng: 01/04/2021

Tù khóa:

giáo dục trực tuyến, dạy học Toán trực tuyến bậc đại học, dạy học Toán truyền thống

Keywords:

online learning, mathematics online learning in higher education, mathemcatics in traditional learning

\section{TÓM TẮT}

Dạy học trực tuyến đã trở nên phổ biến và được áp dụng tại nhiều cơ sở giáo dục đại học ở Việt Nam trong tình hình dịch bệnh hiện nay. Giảng dạy trực tuyến các môn Toán tại các trường đại học đối mặt với nhiều khó khăn hơn so với các môn học khác, vì tính trừu tượng và tư duy logic của môn học. Trong bài báo này, chúng tôi liệt kê các khó khăn riêng biệt từ phía giảng viên và từ phía sinh viên khi tham gia dạy và học trực tuyến các môn Toán trình độ đại học. Bên cạnh đó, chúng tôi đưa ra một số các phương pháp khắc phục các khó khăn trong việc dạy học môn Toán trực tuyến. Chúng tôi xin chia sẻ một số những khó khăn và cách khắc phục trong việc dạy học môn Toán trực tuyến được tiến hành tại Trường Đại học An Giang.

ABSTRACT
Online teaching has rapidly become popular in many higher
education institutions in Vietnam, especially in the current
epidemic situation. Teaching mathematics courses online at
universities is more complicated than other courses because of
these subjects' abstraction and mathematical logic. This article
lists the specific challenges faced by mathematical lecturers and
students in engaging in teaching and learning online mathematics
courses in higher education. We propose methods to overcome
these challenges in teaching mathematics online. We also share
difficulties and solutions in teaching mathematics online
conducted at An Giang University.

\section{Giới thiệu}

Mô hình đào tạo trực tuyến là xu hướng phát triển của giáo dục hiện đại trên thế giới và Việt Nam, do tính linh hoạt, tiện dụng về thời gian và địa điểm của nó. Dạy học trực tuyến giúp giải quyết nhiều vấn đề khó khăn khi người học có thể học mọi lúc mọi nơi, học ở văn phòng, học ở nhà hoặc bất kỳ địa điểm nào thuận tiện và có thể học nhiều lần (Phung, 2018; Tran \& Bui, 2020). Mô hình mới này có thể sử dụng nhiều phần mềm hỗ trợ đắc lực trong quản lý đào tạo, hỗ trợ giải quyết sự phân hóa đến từng đối tượng người học. Đây là điều mà các phương pháp giáo dục đại học truyền thống không thể làm được. 
Tình hình đại dịch Covid-19 đang diễn biến phức tạp, nếu đại dịch bùng phát, để phòng ngừa lây nhiễm dịch bệnh các trường đại học có thể sẽ không cho phép đào tạo tập trung. Cho nên việc phát triển các hệ thống hỗ trợ đào tạo trực tuyến và việc chuẩn bị các nội dung giảng dạy cho giảng dạy trực tuyến là cần thiết trong bối cảnh hiện nay. Trước tình hình thực tiễn này làm phát triển mạnh mẽ và rầm rộ mô hình đào tạo trực tuyến tại các trường đại học.

Các môn Toán học giảng dạy tại các trường đại học có các đặc trưng riêng biệt so với các môn học khác với tính trừu tượng thông qua những suy luận logic chặt chẽ. Các môn toán học trình độ đại học có nội dung phức tạp và chuyên sâu hơn so với các môn Toán bậc học phổ thông. Các môn Toán học tại trường đại học thường được đánh giá là khó hiểu so với các môn học khác vì các môn học này nghiên cứu các cấu trúc trừu tượng như các số, cấu trúc, không gian và các phép biến đổi. So với các môn học khác, khi dạy học các môn Toán bằng hình thức trực tuyến giảng viên cần tăng cường động lực và động cơ học tập cụ thể của sinh viên và khuyến khích sự tham gia và tương tác của sinh viên đối với nội dung môn học (Lin, Tseng, \& Chiang, 2016; Oliver, Kellogg, \& Patel, 2010; Ramasamy, 2009).

So với giáo dục truyền thống, đào tạo trực tuyến có những khuyết điểm riêng như thiếu tương tác trực tiếp, đòi hỏi một động lực học tập rất mạnh mẽ và kỹ năng quản lý thời gian từ các sinh viên và những khó khăn trong việc đảm bảo chất lượng của các hình thức kiểm tra và đánh giá (Ramasamy, 2009; Silalahi, 2020; Tran \& Bui, 2020; Wulantina et al., 2020). Ngoài những khó khăn chung của giáo dục trực tuyến, việc dạy học trực tuyến các môn Toán có những khó khăn đặc thù riêng. Những khó khăn này bao gồm những khó khăn về phía các sinh viên học Toán và khó khăn của giảng viên giảng dạy môn Toán.

Việc dạy học trực tuyến các môn Toán trình độ đại học đã được áp dụng trong các trường đại học trên thế giới đã từ lâu (Ramasamy, 2009; Silalahi, 2020; Wulantina et al., 2020). Tuy nhiên việc dạy học trực tuyến các môn Toán trình độ đại học đã bộc lộ những khuyết điểm nổi bậc và đặc thù so với phương pháp dạy học truyền thống. Ở Việt Nam, chúng ta cũng áp dụng dạy học trực tuyến các môn Toán cho các lớp đào tạo đại học từ xa. Tuy nhiên, chất lượng của các khóa học trực tuyến các lớp đào tạo đại học từ xa này chưa được đánh giá cao. Có ba nguyên nhân chính thúc đẩy chúng tôi làm nghiên cứu này: thứ nhất là khi tiến hành dạy học trực tuyến các môn Toán trình độ đại học, chúng ta sẽ kế thừa và học hỏi những khuyết điểm nổi bậc và đặc thù của các khóa học trực tuyến môn Toán trên thế giới. Thêm vào đó, chúng ta còn có những khó khăn riêng của từng khu vực và từng trường đại học. Thứ hai là khi chuyển sang dạy học trực tuyến các môn Toán trình độ đại học chúng ta cũng phải bảo đảm giữ được chất lượng của khóa học như phương pháp dạy học truyền thống. Thứ ba là việc dạy học trực tuyến bậc đại học ở Việt Nam hiện còn rất non trẻ, trong đó Funix là một trong những trường đại học trực tuyến đầu tiên tại Việt Nam, chính thức thành lập vào tháng 11 năm 2015.

Trong bài viết này, chúng tôi đưa ra những khó khăn riêng biệt của việc dạy học trực tuyến các môn Toán trình độ đại học và các phương pháp khắc phục khó khăn trong việc đào tạo trực tuyến các môn Toán. Chúng tôi cũng trình bày những khó khăn trong việc bước đầu áp dụng hình thức dạy học trực tuyến cho các môn Toán tại Trường Đại học An Giang. Chúng tôi đã áp dụng phương pháp dạy học trực tuyến môn học xác suất thống kê cho 150 sinh viên năm thứ hai, khoa Công nghệ thông tin, Trường Đại học An Giang khóa học 2019-2020. Khóa học hoàn toàn trực tuyến được kéo dài trong năm tuần từ 03/02/2020 đến 06/03/2020. Trong quá trình học trực tuyến chúng tôi tiến hành quan sát và lưu lại khoảng thời gian của từng em tương tác trên cổng học tập trực tuyến. Sau khóa học hoàn toàn trực tuyến này, khi sinh viên trở lại trường sau đợt dịch bùng phát, chúng tôi tổ chức giảng dạy kết hợp giữa dạy học truyền thống và dạy học trực tuyến. Sau khi môn học kết thúc, chúng tôi tiến hành phát phiếu điều tra để đánh giá mức độ hứng thú và tham 
gia bài giảng của các sinh viên.

Bài báo này được chia thành 5 phần. Tiếp theo phần này, Phần thứ 2 sẽ trình bày về những khó khăn riêng biệt của việc dạy học trực tuyến các môn Toán. Kế đến là Phần 3 đề cập đến một số các phương pháp khắc phục các khó khăn trong việc dạy học môn Toán. Phần 4 chúng tôi trình bày về những khó khăn và cách khắc phục trong việc dạy học môn Toán tại Trường Đại học An Giang. Cuối cùng, Phần 5 là kết luận.

\section{Những khó khăn riêng biệt của việc dạy học trực tuyến các môn Toán}

Học tập trực tuyến các môn Toán tại các trường đại học khó hơn việc học trực tuyến các môn học khác bởi vì tư duy logic và tính trừu tượng trong các bài toán cần được hướng dẫn chậm rãi, rõ ràng và thường xuyên lập lại qua nhiều dạng bài tập. Đối với các sinh viên học các môn Toán trực tuyến khó khăn cơ bản của các em là thiếu sự giao tiếp trực tiếp với giảng viên và các bạn cùng lớp. Đối với các giảng viên giảng dạy trực tuyến các môn Toán, việc tăng khối lượng và chất lượng công việc gây nhiều áp lực cho giảng viên (Lin et al., 2016; Oliver et al., 2010; Ramasamy, 2009; Silalahi, 2020; Wulantina et al., 2020).

\subsection{Nhũng khó khăn của sinh viên học các môn Toán}

Sinh viên học Toán cần trao đổi trực tiếp với giảng viên hơn các môn học khác. Dạy học truyền thống các môn Toán mang lại nhiều lợi ích cho sinh viên vì giáo viên có thể hỗ trợ nhiều cho sinh viên hơn so với dạy trực tuyến. Vì toán là môn học khó, sinh viên có thể học được rất nhiều trong quá trình quan sát trực quan giảng viên từng bước giải quyết các bài toán mẫu. Sinh viên cần có thể đặt câu hỏi và nhận được câu trả lời nhanh chóng trong buổi dạy.

Trong khi học trực tuyến sinh viên được khuyến khích tự học. Sự tự lập và thiếu giao tiếp trong quá trình học trực tuyến đôi khi làm các kiến thức Toán, vốn dĩ đã khó, lại càng khó thêm. Đôi khi sinh viên học trực tuyến phải đợi câu trả lời với thời gian lâu hơn và phần giải thích từ giảng viên lại khó hiểu. Điều này cản trở quá trình học tập của sinh viên và làm giảm sự tích cực tham gia vào khóa học của sinh viên.

Ngoài ra sinh viên học trực tuyến các môn Toán còn có nhu cầu trao đổi với các bạn cùng lớp học. Sinh viên học trực tuyến không có nhiều cơ hội trao đổi thông tin với bạn bè cùng khóa học như khi các em học theo phương pháp truyền thống. Phương tiện trao đổi duy nhất của các em là hộp thoại của môn học. Giảng viên sẽ khuyến khích sinh viên đặt câu hỏi và trả lời câu hỏi của các bạn khác trong lớp bằng cách cho điểm chuyên cần cho các hoạt động trao đổi này.

Do nội dung của các môn Toán là trừu tượng và phức tạp hơn các môn học khác, việc các sinh viên học theo nhóm, trao đổi chậm rãi, chi tiết và ngay tại chỗ các nội dung môn học và giải các bài tập liên quan mang lại rất nhiều lợi ích thiết thực và làm cho việc học các môn Toán sẽ dễ dàng hơn rất nhiều. Trong khi đó, sinh viên học trực tuyến thường không nhận được sự trao đổi ngay lập tức. Các trao đổi trên hộp thoại của học trực tuyến thường là các trả lời nhanh và không được chi tiết. Thường các em không đủ kiên nhẫn để viết từng bước hoặc đánh máy từng bước phần giải thích rồi tải lên hộp hội thoại cho các bạn xem.

Sinh viên học trực tuyến các môn Toán tại các trường đại học phải có tinh thần tự lập cao trong học tập. Sinh viên học trực tuyến phải tự phát triển động cơ học tập nhiều hơn so với khi học trên lớp. Sinh viên phải độc lập tìm hiểu các tài liệu giảng dạy, để tự mình xác định được những chỗ còn vướng mắc, những phần nội dung còn thắc mắc. Sinh viên phải chủ động trong việc tìm lời giải, đặt câu hỏi và thúc đẩy quá trình trao đổi với giảng viên và các bạn cũng khóa, cho đến khi chắc chắn rằng mình đã hiểu được nội dung đó. 


\subsection{Nhũ̃ng khó khăn của giảng viên dạy các môn Toán}

Giảng viên phải được đào tạo để sử dụng thuần thục các phần mềm để viết công thức toán và minh họa hình ảnh trực quan trong quá trình giảng dạy online. Để duy trì sự liên tục của cuộc trao đổi trực tuyến khi sinh viên có câu hỏi về một bài toán, giảng viên cần phải đánh từng bước công thức để trình bày bài toán hoặc vẽ hình ảnh trực quan giải thích bài toán trong thời gian ngắn. Vì Toán học có tính trừu tượng rất cao, sinh viên không thể nắm được nội dung khi giảng viên chỉ giải thích mà không trình bày cụ thể và chi tiết từng bước của bài giải. Giảng viên có thể chia sẻ màn hình máy tính của mình khi trao đổi với các sinh viên hoặc có thể quay lại video từng bước thực hiện việc giải bài toán, nếu đó là câu hỏi chung của nhiều em sinh viên.

Giảng viên Toán trực tuyến phải chuẩn bị tài liệu giảng dạy, giáo trình, các video bài giảng và các video thực hành trực tuyến thật chi tiết và cụ thể. Nội dung bài giảng và nội dung bài tập của khóa học phải được thiết kế chi tiết, rõ ràng, dễ hiểu và cẩn thận hơn so với các bài giảng dạy học truyền thống. Giảng viên phải đảm bảo rằng, khi sinh viên cần kiến thức của một phần nội dung nào đó, thì sinh viên sẽ tự tìm được nội dung bài giảng, tài liệu và bài tập liên quan một cách nhanh chóng nhất. Việc thiết kế các nội dung bài giảng và nội dung bài tập cho từng phần của môn học là một khối lượng công việc lớn, và cần sự hợp tác của nhiều giảng viên cho một môn học.

Giảng viên giảng dạy trực tuyến các môn Toán phải thiết kế nhiều hình thức kiểm tra và đánh giá để đảm bảo chất lượng của khóa học trực tuyến. Các hình thức kiểm tra này phải đánh giá được quá trình học tập, tham gia của sinh viên vào khóa học. Các hình thức kiểm tra trực tuyến bao gồm hình thức trắc nghiệm ngắn, hình thức câu hỏi tự luận ngắn, hình thức kiểm tra phỏng vấn trực tiếp và đôi khi giảng viên cần phải kết hợp với hình thức kiểm tra truyền thống. Hình thức trắc nghiệm này trước mỗi bài mới nên được áp dụng. Giảng viên phải xây dựng hệ thống ngân hàng trắc nghiệm ngắn đủ lớn để khuyến khích sinh viên thử sức nhiều lần, cho đến khi các em đạt được điểm mình mong muốn. Các hình thức kiểm tra và đánh giá khác chỉ được thực hiện khi giảng viên đảm bảo được tính trung thực, chính xác và công bằng trong học thuật.

Giảng viên giảng dạy trực tuyến các môn Toán phải khuyến khích và động viên các sinh viên tham gia đặt câu và trao đổi để nắm được nội dung môn học. Giảng viên phải dành nhiều thời gian hơn so với giảng dạy truyền thống để giám sát chặt chẽ quá trình học tập của từng sinh viên bao gồm giám sát chặt chẽ các trao đổi trực tuyến và phản hồi các câu hỏi của sinh viên một cách nhanh nhất. Giảng viên phải đảm bảo việc sinh viên biết là mình đang được giảng viên quan tâm và theo dõi từng nội dung bài học. Giảng viên phải phát hiện và hỗ trợ nhanh chóng và kịp thời khi sinh viên có vấn đề khó khăn về mặt nội dung kiến thức.

\section{Một số phương pháp khắc phục các khó khăn trong việc dạy học môn Toán trực tuyến}

Dựa trên những khó khăn riêng biệt của việc dạy học trực tuyến các môn Toán, chúng tôi xin đưa ra 3 phương pháp khắc phục. Phương pháp thứ nhất là chúng ta nên tăng cường và khuyến khích sự trao đổi và giao tiếp giữa các sinh viên học toán trực tuyến với nhau và với giảng viên. Đối với các lớp học truyền thống, đông sinh viên, khi giáo viên giảng dạy các kiến thức mới, rất ít các sinh viên đặt câu hỏi trực tiếp với giảng viên. Một phần vì các em ngại lớp đông, một phần các em nghĩ là mình có thể trao đổi với các bạn cùng lớp sau hoặc về nhà mình sẽ xem lại và giải quyết được thắc mắc này.

Trong khi dạy trực tuyến các em có thể copy phần câu hỏi và bài giải của mình trao đổi với giảng viên hoặc nhóm hội thoại học tập trực tuyến mà không cần e ngại trình bày trước đám đông. Ngoài phản hồi của giảng viên, đôi khi sinh viên còn nhận được nhiều phản hồi từ bạn cùng lớp khác. Hoặc các bạn cùng lớp có thể học hỏi được kiến thức từ các câu hỏi của các bạn sinh viên khác. 
Tránh các tình huống đối với những kiến thức Toán khó, cộng thêm sự cô lập của sinh viên học toán trực tuyến, làm cho sinh viên cảm giác nội dung môn học càng khó hơn. Điều này ảnh hưởng đến kết quả học tập và sự tích cực tham gia thảo luận của sinh viên. Sinh viên thường thấy quá khó nên không thể hiểu được phần kiến thức này, lạc lối và không thể tham gia trao đổi giao lưu nội dung học này trên nhóm trực tuyến. Trong quá trình thảo luận, giảng viên phải nắm bắt những tình huống như vậy để có sự hỗ trợ và giúp đỡ kịp thời. Để đảm bảo việc trao đổi với giáo viên và các bạn cùng lớp được hồi âm một cách nhanh nhất và chính xác nhất. Giảng viên nên thường xuyên quan tâm, trao đổi cởi mở và khuyến khích sinh viên tham gia vào quá trình học. Phải để sinh viên có cảm giác an tâm là quá trình học tập của mình được hỗ trợ và giám sát chặt chẽ.

Phương pháp thứ hai là chúng ta nên đẩy mạnh đầu tư vào nguồn tài liệu trực tuyến bao gồm các video bài giảng, giáo trình chính, hệ thống bài tập và hệ thống các bài kiểm tra và đánh giá. Sinh viên học Toán trực tuyến cần nội dung giảng dạy cụ thể, rõ ràng và chi tiết. Giảng viên phải đầu tư vào chất lượng bài giảng videos vừa ngắn gọn và thu hút vừa dễ hiểu. Đối sinh viên online việc giao tiếp với giảng viên và giáo viên bị hạn chế, nên đối với những kiến thức mới và khó, nhóm giảng viên nên nghiên cứu trình bày bằng các videos bài giảng một cách ngắn gọn và dễ hiểu. Phải đảm bảo rằng khi sinh viên xem xong một bài giảng videos ngắn, sinh viên phải biết các khái niệm để tự mình giải cơ bản các bài tập Toán được cho phía sau phần lý thuyết đó.

Giảng viên phải thiết kế nhiều hình thức kiểm tra và đánh giá để đảm bảo chất lượng của khóa học trực tuyến. Các hình thức kiểm tra đánh giá này một phần để kiểm tra và đánh giá quá trình học tập của sinh viên, một phần khác khuyến khích sinh viên học tập và phát hiện những lỗ hỏng kiến thức của mình. Sau khi xem lại phần kiến thức mình thiếu sót, sinh viên có thể thử lại bài kiểm tra được chọn ngẫu nhiên trong hệ thống, sinh viên làm như vậy đến khi nào đạt được điểm mình mong muốn. Các hình thức kiểm tra và đánh giá phải đảm bảo được tính trung thực, chính xác và công bằng trong học thuật.

Phương pháp thứ ba là tăng cường tính tự giác của sinh viên khi học Toán trực tuyến để khắc phục những khó khăn trong các khóa học Toán trực tuyến. Giảng viên cần phải phát hiện ra những lỗ hỏng kiến thức của sinh viên qua các câu hỏi trực tuyến của sinh viên. Sẽ có rất nhiều sinh viên không xem bài giảng hoặc đọc giáo trình trước, thay vì bắt đầu ngay vào việc giải các bài tập. Khi phát hiện ra các tính huống này, giảng viên cần hướng dẫn sinh viên quay lại để xem bài giảng và đọc giáo trình ở các phần cụ thể, trước khi tiến hành giải các dạng bài tập cụ thể.

Công việc chính của các giảng viên dạy các môn Toán trực tuyến là khuyến khích sinh viên đọc và xem bài giảng và khuyến khích sinh viên đặt các câu hỏi nhiều như có thể. Cho đến khi sinh viên chắc chắn hiểu rõ phần kiến thức Toán đó.

\section{Những khó khăn và cách khắc phục trong việc dạy học môn Toán tại Trường Đại học An Giang}

Trường đại học An Giang đã và đang phát triển hệ thống hỗ trợ đào tạo trực tuyến thông qua cổng học tập Cohota dưới sự hỗ trợ xây dựng và quản lý của khu công nghệ phần mềm Trường Đại học Quốc gia thành phố Hồ Chí Minh. Sự linh hoạt và dễ sử dụng của phần mềm này đã hỗ trợ rất nhiều cho quá trình dạy và học của giảng viên và sinh viên. Tuy nhiên, vì việc triển khai đào tạo trực tuyến trong một thời gian ngắn, nên không tránh khỏi những khó khăn và hạn chế vốn có của hình thức giáo dục trực tuyến. Một trong những khó khăn lớn nhất là phần lớn giảng viên và sinh viên của trường chưa được làm quen với hình thức kiểm tra và đánh giá trực tuyến. Việc này có thể ảnh hưởng đến chất lượng của cả khóa học. 
Khi đại dịch bùng phát vào đầu năm 2020, Trường Đại học An Giang đã đóng cửa để phòng chống dịch trong khoảng thời gian hơn hai tháng sau tết âm lịch năm 2020. Các giảng viên được khuyến khích chuyển các nội dung môn học sang hình thức dạy học trực tuyến. Giảng viên dạy học các môn Toán, đã tiến hành chuyển bài giảng của mình sang các video bài giảng và đăng tải trên hệ thống đào tạo trực tuyến của trường. Sau đây là khảo sát tính hiệu quả của việc dạy trực tuyến môn xác suất thống kê cho 150 em sinh viên năm hai Trường Đại học An Giang. Khoảng $80 \%$ các em đã đăng nhập và tải các giáo trình và các video bài giảng về. Trong số đó chỉ có khoảng 50\% các em xem toàn bộ video bài giảng. Phần còn lại hệ thống Cohota chỉ ghi nhận được các em có đăng nhập và xem qua các tài liệu học tập trong khoảng từ 10 đến 20 phút. Trong trường hợp này chúng ta không đánh giá được là các em tải các tài liệu trực tuyến về và học trên máy tính riêng hay các em chỉ dừng lại tại đó.

Sau khi đợt dịch tạm lắng ở Việt Nam, các trường đại học mở cửa trở lại. Các em sinh viên quay trở lại học dưới hình thức truyền thống. Giảng viên kết hợp giảng dạy trên lớp và cung cấp các tài liệu trực tuyến và mở hộp thoại trao đổi trực tuyến giữa các sinh viên và giảng viên. Qua khảo sát trả lời của các em sinh viên, có khoảng $80 \%$ các em sinh viên thấy các video bài giảng là rất có ích khi các em vừa lên lớp học vừa có các video bài giảng để xem ở nhà. Các em có thể xem lại bài giảng trong trường hợp không thể lên lớp hoặc trên lớp các em theo dõi nội dung bài giảng không kịp. Thông qua hộp hội thoại thông tin trao đổi giữa giảng viên và sinh viên và giữa các sinh viên với nhau nhanh và chính xác hơn việc sử dụng email như trước đây. Việc trao đổi nội dung bài học trên hộp hội thoại giữa các sinh viên là có nhưng chưa được nhiều. Vì kết hợp với giảng dạy truyền thống, nên các em sinh viên vẫn thích trao đổi với nhau trên lớp, sau các bài giảng hơn là trên hộp hội thoại chung.

Vì Toán học là các môn học có nội dung khó, nên hiện nay cả sinh viên và giảng viên đều lựa chọn phương pháp dạy học truyền thống thay vì giảng dạy trực tuyến. Tuy nhiên đội ngũ giảng viên đã chuẩn bị sẵn sàng các tài liệu online, videos bài giảng và hệ thống ngân hàng câu hỏi trên cơ sở đó xây dựng hệ thống ngân hàng trắc nghiệm ngắn trong trường hợp nhà trường có kế hoạch chuyển sang giảng dạy trực tuyến.

\section{Kết luận}

Các môn Toán học giảng dạy tại các trường đại học thường có tính trừu tượng và tính tư duy logic phức tạp hơn các môn học khác. Cho nên khi chuyển sang hình thức giáo dục trực tuyến, việc giảng dạy các môn Toán ở bậc đại học lại có những khó khăn riêng mang tính đặc trưng của môn học. Việc giảng dạy các môn Toán bậc đại học bằng phương pháp trực tuyến nên được tiến hành chậm hơn so với các môn học khác, khi mà sinh viên và giảng viên đã quen dần với hình thức học trực tuyến. Việc giảng dạy các môn Toán bậc đại học bằng phương pháp trực tuyến là khả thi, và chất lượng của khóa học trực tuyến có thể được đảm bảo như ở các lớp học truyền thống. Tuy nhiên, nhà trường cần phải đầu tư hỗ trợ về mặt kỹ thuật cho các giảng viên khi tham gia giảng dạy trực tuyến. Nhà trường cần phải quan tâm và khuyến khích giảng viên từng bước tiến hành chuyển đổi nội dung giảng dạy từ hình thức truyền thống sang trực tuyến. Vì chuẩn bị cho việc giảng dạy các môn Toán bằng hình thức trực tuyến thực sự làm tăng tăng khối lượng công việc của giảng viên lên rất nhiều lần so với dạy truyền thống.

\section{Tài liệu tham khảo}

Lin, Y. W., Tseng, C. L., \& Chiang, P. J. (2016). The effect of blended learning in mathematics course. EURASIA Journal of Mathematics, Science and Technology Education, 13(3), 741-770. 
Oliver, K., Kellogg, S., \& Patel, R. (2010). An investigation into reported differences between online math instruction and other subject areas in a virtual school. Journal of Computers in Mathematics and Science Teaching, 29(4), 417-453.

Phung, N. X. (2018). Giáo dục Việt Nam trong bối cảnh cuộc cách mạng công nghiệp lần thứ tư [Vietnamese education in the context of the fourth industrial revolution]. Retrieved December 10, 2020, from Vinh University website: http://vinhuni.edu.vn/van-ban/seo/giao-duc-vietnam-trong-boi-canh-cuoc-cach-mang-cong-nghiep-lan-thu-tu-86324

Ramasamy, R. (2009). Mathematics in online-learning: The difference in its approach compared to face-to-face teaching. In ICI9-International conference on information, Kuala Lumpur, Malaysia, (pp. 33-39). Retrieve December 5, 2012, from http://library.wou.edu.my/ vertical/vf2009-17.pdf.

Silalahi, P. (2020). Design and development blended learning approach for student low achievement in mathematics. In First International Conference on Applied Science and Technology (iCAST 2018) (pp. 61-64). France, Paris: Atlantis Press.

Tran, T. Q., \& Bui, H. V. (2020). Quản lý dạy học trực tuyến trong các trường đại học kỹ thuật tại thành phố Hồ Chí Minh [Management of online teaching in technical universities in Ho Chi Minh City]. Tạp chí Khoa học Xã hội - Đại học Mở Thành phố Hồ Chí Minh, 15(1), 46-53.

Wulantina, E., Ikashaum, F., Mustika, J., Merliza, P., Loviana, S., Rahmawati, N. I., \& Andianto, A. (2020). Self-efficacy of mathematics students' on e-learning during covid 19 pandemic. In Proceeding international webinar on education 2020 (pp. 109-116). Indonesia: Beranda. 